\title{
Neonatal screening for severe combined immunodeficiency caused by an adenosine deaminase defect: $A$ reliable and inexpensive method using tandem mass spectrometry
}

\author{
Chiara Azzari, MD, PhD, ${ }^{\mathrm{a}, \mathrm{b}}$ Giancarlo la Marca, PharmSc, ${ }^{\mathrm{b}, \mathrm{c}}$ and Massimo Resti, MD ${ }^{\mathrm{a}, \mathrm{b}}$ Florence, Italy
}

Background: Adenosine deaminase (ADA)-severe combined immunodeficiency (SCID) is an SCID caused by a defect in the enzyme adenosine deaminase. It is usually fatal in infancy because of severe recurrent infections. When diagnosis is made,

permanent damage caused by infections or by metabolites is often present. Gene therapy, bone marrow transplantation, or enzyme therapy might be effective if performed early. ADA-SCID complies with all the criteria for inclusion in a newborn screening program. However, screening methods are still expensive or provide a non-negligible number of indeterminate results.

Objective: The aim of the present study was to develop a simple, reliable, and inexpensive method for diagnosis of ADA-SCID by using dried blood spot (DBS) samples taken at birth. Cost per test was calculated, including the cost for reagents, equipment, and operators.

Methods: DBS samples from 4 patients with genetically confirmed ADA-SCID and 12,020 DBS samples from healthy newborns were examined. Adenosine and 2'-deoxyadenosine were tested by using tandem mass spectrometry (PCT EP2010/ 070517).

Results: The mean levels of adenosine and 2 '-deoxyadenosine were 7.8 \pm 3.1 and $8.5 \pm 6.0 \mu \mathrm{mol} / \mathrm{L}$, respectively, in affected children; adenosine was found at $0.23 \pm 0.09 \mu \mathrm{mol} / \mathrm{L}$, whereas 2 '-deoxyadenosine was never detected in healthy control subjects (adenosine: $P<10^{-6}[95 \%$ confidence limit, 7.59-7.78] and $2^{\prime}$-deoxyadenosine: $P<10^{-6}$ [95\% confidence limit, 8.65-8.82] for control subjects vs patients with ADA-SCID). No indeterminate or false-positive results were found. Cost per test was $€ 0.01$ (\$0.013). A pilot population-based newborn screening for ADA-SCID has started in Tuscany, Italy.

Conclusion: Tandem mass spectrometry can be used for diagnosis of one of the most frequent form of SCID at a negligible cost. (J Allergy Clin Immunol 2011;127:1394-9.)

Key words: Adenosine deaminase, severe combined immunodeficiency, newborn screening, tandem mass spectrometry

\footnotetext{
From the Departments of ${ }^{\mathrm{a}}$ Pediatrics and ${ }^{\mathrm{c}}$ Pharmacology, University of Florence, and ${ }^{\mathrm{b}}$ Anna Meyer Children's University Hospital, Florence.

All of the authors contributed equally to this work.

Supported in part by a donation from Famiglia Cassigoli (C.A.) and by a grant from the University of Florence (C.A. and G.I.M.) and Anna Meyer Children's University Hospital (M.R.) and the Tuscany (Italy) region (C.A., G.l.M., and M.R.).

Disclosure of potential conflict of interest: G. La Marca receives research support from the Tuscany Region, Italy. The rest of the authors have declared that they have no conflict of interest.

Received for publication January 4, 2011; revised February 18, 2011; accepted for publication March 24, 2011.

Reprint requests: Chiara Azzari, MD, PhD, Department of Pediatrics, University of Florence, Anna Meyer Children's University Hospital, Viale Pieraccini, 24, 50139 Firenze, Italy. E-mail: chiara.azzari@unifi.it.

$0091-6749 / \$ 36.00$

(C) 2011 American Academy of Allergy, Asthma \& Immunology

doi:10.1016/j.jaci.2011.03.040
}

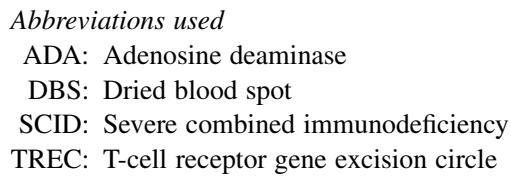

Severe combined immunodeficiency (SCID) is a group of severe diseases that affect the immune system. Infants with SCID are healthy at birth but die of severe infections in infancy unless adequate therapy is provided. ${ }^{1-3}$ Unfortunately, almost all infants with SCID are not identified in the preinfection period; when the diagnosis is hypothesized, usually a severe infection has just occurred. At that time, however, even though a correct therapeutic intervention is started, damages caused by the severe infections (eg, meningitis, encephalitis, and severe pneumonia) can already be present, ${ }^{1,3}$ and permanent sequelae can be an important burden both for patients and families, as well as for the society. Moreover, cases in which children die of severe infection before diagnosis of SCID is made are not infrequent.

Adenosine deaminase (ADA) deficiency is a fatal autosomal recessive form of SCID caused by an inherited disorder of purine metabolism. ${ }^{4}$ ADA is an enzyme of the purine salvage pathway that catalyzes the deamination of adenosine and 2 '-deoxyadenosine to inosine and 2 -deoxyinosine, respectively. In the typical form (neonatal onset, $85 \%$ to $90 \%$ ) of ADA deficiency, absence of the enzyme and accumulation of toxic metabolites are present. The classical phenotype includes a severe defect of the immune system with the absence of both cellular and humoral immunity and permanent damage to other organs and systems, such as the brain or liver. ${ }^{4,5}$ In these cases ADA-SCID is usually fatal within the first months of life in untreated patients and is associated with severe sequelae in those who are treated late. Unlike many other forms of SCID, the systemic nature of ADA expression also results in nonimmunologic abnormalities in a significant proportion of children who might have neurodevelopmental defects, sensorineural deafness, and/or skeletal abnormalities. ${ }^{6}$ Systemic manifestations are frequent, and their severity is proportionate to blood and urine levels of metabolites and to age at diagnosis. The incidence of ADA deficiency is estimated to be between 1 in 375,000 and 1 in 660,000 live births ${ }^{4}$ and accounts for around $15 \%$ to $20 \%$ of all SCID cases. However, the incidence of the disease is probably underestimated because of early deaths caused by infections and might be higher in specific ethnic groups worldwide, especially where the rate of consanguinity is high. ${ }^{7}$ Enzyme replacement therapy, hematopoietic stem cell transplantation, or gene therapy might be curative, but their efficacy is dependent on the early approach. ${ }^{8}$ Whichever therapy is chosen, it should be started as soon as possible after birth to obtain the best therapeutic effect through a rapid metabolic detoxification. In children 
undergoing transplantation, the survival curve is inversely proportional to the age when transplantation is done., ${ }^{4,8}$

At present, diagnostic methods are still aimed to uncover evidence of ADA toxic metabolites in urine or a defect in ADA activity in blood obtained by means of venipuncture. ${ }^{9}$ Other methods have been described, but most of them have in common the application in children in whom the diagnosis is already hypothesized and clinical symptoms are already present. On the contrary, affected infants should receive diagnoses before the onset of infection to maximize the opportunity for life-saving treatment. Therefore a population-based newborn screening program could be the correct approach.

It has been demonstrated that the absence of T-cell receptor gene excision circles (TRECs) could identify infants with SCID by using DNA from dried blood spot (DBS) samples collected for screening newborns ${ }^{1}$; however, as described by the authors, those methods still have a non-negligible number of indeterminate tests and a cost ( $>\$ 5$ per test) that might be unaffordable in many countries.

Expanded newborn screening by means of tandem mass spectrometry has grown from the analysis of a few tens of thousands of samples from Western Pennsylvania in 1992 to nearly 4 million in the United States in 2008 and millions more throughout the world. ${ }^{10}$ In Tuscany (Italy), officially mandated by a legislative action, the program has screened all babies born since November 2004 (approximately 40,000/y) for selected acylcarnitines and amino acids. ${ }^{11}$

The aim of the present work was to set up a simple and inexpensive method of working with DBS samples taken at birth for other newborn screening to detect ADA metabolites with high sensitivity and specificity.

\section{METHODS}

\section{Guthrie card collection procedure}

DBS samples are routinely collected from all neonates born in Tuscany (about 40,000/y). Blood collection (4 DBSs on a Guthrie card) is recommended for between 48 and 72 hours of life. Blood samples are obtained by means of heel stick, spotted on filter paper (903; Whatman GmbH, Dassel, Germany), dried, and sent daily by courier to the central newborn screening laboratory. An expanded newborn screening is routinely performed, including hypothyroidism, cystic fibrosis, biotinidase deficiency, and more than 40 inborn errors of metabolism of amino acids, organic acids, and fatty acids.

All Guthrie card are analyzed within 2 days from collection; after use, all cards are stored and, if not used, discarded after 10 years.

\section{Tandem mass spectrometry}

An ABI SCIEX (Toronto, Canada) API 2000 bench-top Triple-Quad Mass Spectrometer equipped with the turbo ion spray source was used for this study. The turbo ion spray source operated under positive ion mode at a voltage of $5500 \mathrm{~V}$ and a "turbo" gas flow of $10 \mathrm{~L} / \mathrm{min}$ of air heated to $350^{\circ} \mathrm{C}$ (nominal heating-gun temperature).

Mass calibration and resolution adjustments on the resolving quadrupoles were performed automatically by using a polypropylene glycol $10^{-7} \mathrm{~mol} / \mathrm{L}$ solution introduced through the built-in infusion pump. The resolution was set on both resolving quadrupoles at 0.7 atopic mass units (measured at one half height) for all mass spectrometric and tandem mass spectrometric experiments.

Collision-activated dissociation tandem mass spectrometry was performed through the LINAC Q2 collision cell, operating with 10 milliTorr pressure of nitrogen as collision gas.

Declustering potential, collision exit potential, and collision energy were automatically optimized for adenosine, deoxyadenosine, ribose- $1-{ }^{13} \mathrm{C}$ - adenosine, and ${ }^{13} \mathrm{C}_{5}$ deoxyadenosine by using the quantitation optimization option. the resulting declustering potential was $+70 \mathrm{~V}$. Optimal collision energy and collision exit potential were found at 26 and $20 \mathrm{~V}$, respectively.

Mass spectrometric and tandem mass spectrometric spectra were collected in continuous-flow mode by connecting the infusion pump directly to the turbo ion spray source. Standard solutions of $1 \mathrm{ng} / \mathrm{mL}$ adenosine, deoxyadenosine, ribose-1- ${ }^{13} \mathrm{C}$-adenosine, and ${ }^{13} \mathrm{C}_{5}$ deoxyadenosine in an aqueous solution of $50 \%$ methanol containing $0.1 \%$ formic acid were infused at $10 \mu \mathrm{L} / \mathrm{min}$. From these experiments, the resulting ion-pair transitions for the quantitative experiment are as follows: $268.2>136.1$ and $269.2>136.1$ for adenosine and ribose- $1-{ }^{13} \mathrm{C}$-adenosine, respectively, and $252.2>136.1$ and $257.2>136.1$ for deoxyadenosine and ${ }^{13} \mathrm{C}_{5}$ deoxyadenosine, respectively.

\section{Guthrie cards from patients with ADA-SCID and control subjects}

DBS samples from 4 patients with genetically confirmed early-onset ADASCID (1 month, 4 months, 4 years, and 6 years old, respectively) were evaluated. Three of them were born and resident in Tuscany, and the forth was born in a different region but referred to our hospital for immunologic evaluation. DBS samples had been obtained as previously described and tested for individuation of ADA metabolites with an API 2000 Tandem Mass Spectrometer (Applied Biosystems, Foster City, Calif).

As controls, DBS samples from 12,020 healthy newborns (10,960 full-term and 1,060 premature infants weighing $<1,500 \mathrm{~g}$ ) from the same region and collected in the years 2005 to 2009 were also analyzed. Informed consent was obtained from parents or guardians. The study was approved by the local institutional review board.

\section{Sample preparation}

Sample preparation for routinely screening tests is exactly as reported by la Marca et $\mathrm{al}^{12}$ in 2008 , with the exception of the butylation procedure, which is not carried out.

\section{Method validation}

The method for individuating ADA metabolites on DBS samples has been patented (PCT EP2010/070517). The method provides quantitative analysis of different metabolites, among them adenosine and deoxyadenosine. The cutoff of the test is $0.005 \mu \mathrm{mol} / \mathrm{L}$ for any of the 2 analytes.

Reference standard blood (whole blood) spots were prepared by using a pooled whole-blood sample obtained from 5 subjects. The blood was processed by adjusting the hemoglobin concentration to $17 \mathrm{mg} / \mathrm{dL}$ and adding adenosine and deoxyadenosine at known concentrations. The processed blood was dispensed onto filter paper cards to form blood spots on the filter paper matrix. Each blood spot was generated by dispensing $25 \mu \mathrm{L}$ of processed blood. The blood spots were allowed to dry overnight.

A small disc $(3.2 \mathrm{~mm})$ of a DBS was punched and deposited in a well of a microwell plate. The sample was extracted by dispensing $300 \mu \mathrm{L}$ of an extraction solution consisting of a mixture of methanol and aqueous solution of $3 \mathrm{mmol} / \mathrm{L}$ hydrate hydrazine at an approximate relative volume/volume ratio of $66.6 \%$ and $33.3 \%$, respectively. Internal standards, stable heavy isotope analogs of several amino acids, carnitine, acylcarnitines, succinylacetone, adenosine, and deoxyadenosine, were also present in the extract solution.

The extracted sample was injected into an electrospray triple quadrupole tandem mass spectrometer with the aid of an automated liquid-handling device. Mass spectral data for the amino acids were acquired through a neutral loss scan of $46 \mathrm{Da}$; mass spectral data for the acylcarnitines were acquired through a precursor ion scan of $85 \mathrm{Th}$. Mass spectral data for adenosine, deoxyadenosine, and succynilacetone were acquired through multiple-reaction monitoring. The percentage of each analyte recovered was determined through comparison with an internal standard for each analyte.

The imprecision of the assay was determined by analyzing the samples described in Table I. Each sample run consisted of sextuplicate punches of each sample, which were processed and measured as described. The study 
included 6 such runs a day for a total of 6 days. With this information, the imprecision components of within run, between run/within day, and between day were determined, from which the total imprecision was determined. The results of the imprecision analysis of adenosine and deoxyadenosine are shown in Table I.

\section{Cost analysis}

Costs for reagents, equipment, and laboratory operators have been considered to evaluate the total cost of the test per patient. Costs of Guthrie cards and transport to the newborn screening laboratory were not included because the test was performed on the same Guthrie card already used for all other neonatal screening routinely performed in Tuscany.

\section{RESULTS}

\section{Sensitivity and specificity of the test}

All the DBS samples obtained in the first days of life from the 4 patients with ADA-SCID demonstrated high levels of adenosine and $2^{\prime}$-deoxyadenosine. In the patients the mean \pm SD levels of adenosine and $2^{\prime}$-deoxyadenosine were $7.8 \pm 3.1$ and $8.5 \pm 6.0$ $\mu \mathrm{mol} / \mathrm{L}$, respectively, and ranged between 4.4 and $11.8 \mu \mathrm{mol} / \mathrm{L}$ for adenosine and 2.5 and $16.2 \mu \mathrm{mol} / \mathrm{L}$ for $2^{\prime}$-deoxyadenosine (Fig 1). 2'-Deoxyadenosine was never detected in any of the 12,020 healthy control samples, whereas adenosine had a mean \pm SD level of $0.23 \pm 0.09 \mu \mathrm{mol} / \mathrm{L}$ and ranged between 0.01 and $0.81 \mu \mathrm{mol} / \mathrm{L}$, with a median of $0.21 \mu \mathrm{mol} / \mathrm{L}(P<$ $10^{-6} ; 95 \%$ confidence limit, 7.59-7.78 vs patients with ADASCID) and no difference between full-term or premature infants. No false-positive or indeterminate test results were found.

As for adenosine, the ratio between the mean level in children with ADA-SCID and control subjects was 34.0, whereas for 2 -deoxyadenosine, the ratio was incalculable because of the absence of 2 -deoxyadenosine in healthy subjects. However, hypothesizing a mean level equal to the cutoff $(0.005 \mu \mathrm{mol} / \mathrm{L})$ in healthy newborns, the ratio between children with ADASCID and healthy children would be 1700 for that analyte $\left(P<10^{-6} ; 95 \%\right.$ confidence limit, $8.65-8.82$ vs patients with ADA-SCID).

\section{Costs per patient}

No extra operator time was necessary for sample preparation or sample analysis beside the time necessary for routine neonatal screening. Similarly, no instrumentation was necessary beside that routinely used for neonatal screening, and the instruments were not used for longer times. Therefore the cost was calculated for reagents only. The cost was $€ 0.006$ per patient ( $=\$ 0.0078$ on September 22, 2010). Reagent waste during dilution, aliquot preparation, and sampling is usually unavoidable, and therefore the cost per patient was rounded up to $€ 0.01$ ( $\$ 0.013)$.

\section{DISCUSSION}

The results of the present study demonstrate that patients with ADA-SCID can easily have their disease diagnosed at birth by using the same method (tandem mass spectrometry) already used for neonatal screening and with a cost of $€ 0.01$ per patient.

As with other SCIDs, patients with ADA-SCID have severe and often fatal infections if they remain untreated and might have permanent sequelae if treated late. Clinical features of ADASCID are those that are considered necessary to include a disease
TABLE I. Results of the imprecision assay for adenosine and deoxyadenosine detection in DBS samples: imprecision within run, between runs, within day, and between days

\begin{tabular}{|c|c|c|c|c|c|}
\hline $\begin{array}{l}\text { Investigated } \\
\text { metabolite }\end{array}$ & $\begin{array}{c}\text { Spiking } \\
\text { (nmol/L) }\end{array}$ & $\begin{array}{c}\text { Intraday } \\
\text { precision } \\
(\%), n=6\end{array}$ & $\begin{array}{c}\text { Interday } \\
\text { precision } \\
(\%), n=6\end{array}$ & $\begin{array}{l}\text { Average } \\
\text { readings } \\
\text { (nmol/L) }\end{array}$ & $\begin{array}{c}\text { Accuracy } \\
(n=6)\end{array}$ \\
\hline Adenosine & 0 & 0 & 0.0 & 0.0 & \\
\hline Adenosine & 33 & 3.5 & 3.1 & 34.0 & 103.1 \\
\hline Adenosine & 165 & 4.9 & 3.7 & 158.0 & 95.8 \\
\hline Adenosine & 330 & 7.8 & 6.0 & 336.2 & 101.9 \\
\hline Adenosine & 3300 & 3.8 & 4.8 & 3299.7 & 100.0 \\
\hline Adenosine & 6600 & 2.1 & 2.6 & 6594.2 & 99.9 \\
\hline Adenosine & 9900 & 2.3 & 2.0 & 9899.9 & 100.0 \\
\hline Deoxyadenosine & 0 & 0 & 0.0 & 0.0 & \\
\hline Deoxyadenosine & 33 & 19.6 & 16.9 & 32.8 & 92.9 \\
\hline Deoxyadenosine & 165 & 6.6 & 4.8 & 169.6 & 100.3 \\
\hline Deoxyadenosine & 330 & 5.2 & 3.6 & 325.4 & 100.6 \\
\hline Deoxyadenosine & 3300 & 5.6 & 6.7 & 3300.2 & 100.3 \\
\hline Deoxyadenosine & 6600 & 3.4 & 3.4 & 6599.2 & 100.0 \\
\hline Deoxyadenosine & 9900 & 3.1 & 3.1 & 9904.9 & 100.0 \\
\hline
\end{tabular}

in a neonatal screening program: high mortality and morbidity of the disease, which could be cured if recognized early, and the availability of a simple and inexpensive test able to individuate disease markers.

Recently, a statement of the Advisory Committee on Heritable Disorders in Newborns and Children ${ }^{13}$ has underlined the importance of screening programs for immunodeficiencies.

As suggested, ${ }^{1}$ progressing from conception to implementation of neonatal screening requires positive answers to many questions regarding sensitivity and specificity, performance for large-scale use, and possible limitation to a special subpopulation. Indeed, although screening must be highly sensitive not to miss all true cases, specificity is also very important to avoid expenses associated with unneeded follow-up.

The method we describe, even though performed as a pilot study in 4 patients with ADA-SCID and in a limited group of about 12,000 healthy control subjects, seems to be promising. First, it is able to detect the analyte without overlapping between newborns with ADA-SCID and control subjects, with mean values in patients that were more than 30 times higher for adenosine and more than 1,700 times higher for $2^{\prime}$-deoxyadenosine. Moreover, the test had no false-positive results, and the large difference in the measured ranges between patients and control subjects makes it useless to use any algorithm to predict true positivity. The absence of follow-up visits or blood examinations because of false-positive results further increases cost-effectiveness.

ADA-SCID screening by means of tandem mass spectrometry from DBS samples could be easily included in a population-based screening program because of its low cost and because it does not require extra instrumentation or extra time for operators already working in screening programs. This is an important point because policymakers consider, ${ }^{14}$ as a primary topic, whether a sufficient public health infrastructure exists to support the new test. Actually, different from screening based on TRECs, which requires a laboratory with the ability to perform quantitative PCR (a technique that currently is not used in most newbornscreening programs), ${ }^{15}$ screening based on tandem mass spectrometry can be immediately performed in laboratories working on newborn screening. 


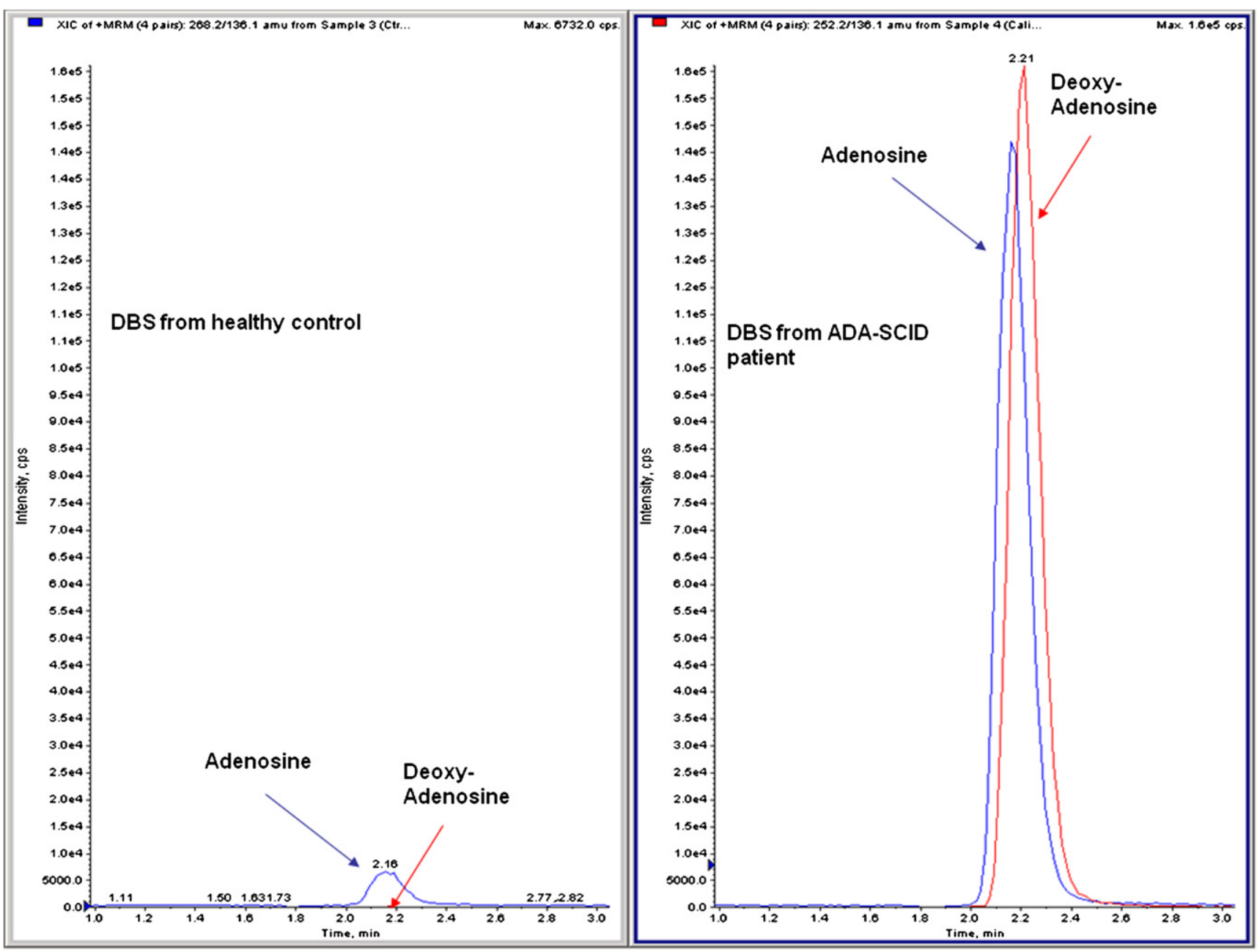

FIG 1. Histograms of metabolite (adenosine and 2 '-deoxyadenosine) concentration in a patient with ADA-SCID and a representative control subject. Adenosine and 2'-deoxyadenosine levels of a patient with ADA-SCID (right) and a representative healthy control subjects (left), as obtained from DBS samples taken at birth, are shown. Adenosine is shown in blue, and 2'-deoxyadenosine is shown in red.

The method is applicable also to premature infants whose mean levels of adenosine or $2^{\prime}$-deoxyadenosine do not differ from those found in full-term babies. None of the patients with ADA included in our study were born prematurely; however, it is well known that high levels of toxic metabolites are already present during fetal life, ${ }^{16}$ and low numbers of T lymphocytes and $2^{\prime}$-deoxyadenosine triphosphate accumulation were found in the blood of affected fetuses at 18 weeks' gestation. ${ }^{16}$

ADA deficiency can sometimes manifest with partial enzyme deficiency, with immunologic symptoms in adulthood, or with no symptoms at all. We could not test neonatal blood spots obtained from patients with adult-onset ADA-related immunodeficiency to evaluate whether our test, which is performed at birth, can also individuate this subpopulation of abnormal patients with ADA. However, we could imagine 3 different hypotheses.

The first hypothesis involves patients in whom enzyme activity is reduced and partial accumulation of metabolites is present. This situation seems to be the most frequent in patients with partial ADA deficiency. ${ }^{17-19}$ Metabolite accumulation is present even in patients who are still asymptomatic, although at lower levels compared with that seen in patients with complete ADA deficiency. This situation is well known for other metabolic diseases routinely screened at birth all over the world, such as hyperphenylalaninemias. In the case of ADA deficiency, individuation of partial metabolite accumulation would be even easier because in healthy subjects the metabolite $2^{\prime}$-deoxyadenosine is completely absent. Therefore its presence, even at very low blood levels, would suggest a partial enzyme inactivity. Moreover, patients with late-onset ADA-SCID have been reported to have $2 '$-deoxyadenosine levels that are at least 2-log higher than in healthy control subjects, ${ }^{18}$ and therefore they could be easily found.

The second hypothesis concerns patients with partial ADA inactivity who retain absolutely normal detoxification ability. ${ }^{19}$ These patients might remain asymptomatic for all their lives and be identified only by population screening of healthy adults. We can imagine that if detoxification activity remains complete and stable for all their lives, neither tandem mass spectrometric methods nor methods based on TRECs can detect these subjects; however, because they remain asymptomatic, to individuate them 
would be beyond the aims of a neonatal screening for inherited disease.

The third hypothesis involves patients with completely normal enzyme activity at birth, decreasing over time and resulting in the phenotype of immunodeficiency later in life. This hypothesis seems the most unlikely; to our knowledge, serial studies from birth to adulthood have not been performed in patients with adult-onset ADA deficiency. However, even if these patients could not be given diagnoses at birth because of the presence of a complete metabolite detoxification, it would be easy to make a diagnosis using the same tandem mass spectrometric method and at the same cost later in life as soon as symptoms suggest the disease.

A trial for a population-based neonatal screening in Tuscany was started in September 2010 to confirm the results of a larger population and to perform early diagnosis of ADA-SCID.

Different from tests based on quantitation of TRECs, which might provide indeterminate results because of poor DNA recovery, ${ }^{1}$ the method described in the present research does not seem to be affected by long-term storage or bad storage conditions. Actually, even after 6 years, ADA metabolites are still present at high levels, so that the method could unequivocally individuate a patient with ADA-SCID born in 2004.

Different methods for measurement are used in clinical practice, ranging from HPLC ${ }^{20}$ to capillary electrophoresis ${ }^{21}$ and even reverse-phase HPLC with electrospray ionization tandem mass spectrometry. $^{22}$

However, all these methods are applied on urine samples and are used when a clinical suspicion of immunodeficiency has already been formulated because of the onset of severe infections. All of them are time consuming and therefore not applicable for screening purposes. In fact, we evaluated that the liquid chromatographic-mass spectrometric method ${ }^{23}$ requires not less than 20 minutes per sample. Moreover, urine is not available as a biological matrix for screening purposes at birth.

ADA-SCID seems to have an incidence of $1: 375,000$ to $1: 660,000^{4}$; by using the method described, the cost for individuating 1 patient would be $€ 3,750$ to $€ 6,600$, and that amount is probably lower than the cost of hospitalization of a patient with ADA-SCID for his or her first infection. Indeed, the cost for hospitalization in Italy is more than $€ 500$ per day in pediatric units and more than $€ 1000$ per day in intensive care units. ${ }^{24}$ Moreover, it should be considered that the incidence of ADA-SCID might be greater, being underestimated because of early death from infective disease or disorders caused by toxic metabolites. Actually, at least 3 patients with ADA-SCID were born and resident in Tuscany during the study period (2004-2009); given that in the same period in Tuscany the number of live birth was $158,695,{ }^{25}$ the incidence of ADA-SCID in Tuscany seems to be 1:53,000 or higher, more than previously thought. ${ }^{4}$ This cannot be explained by the high rate of consanguinity, which, on the contrary, is rare in Tuscany.

Cost-effectiveness analysis was out of the aim of the present study; however, the extremely low cost per test suggests that this test could be a worthwhile addition to newborn screening panels. It has been recently reported in a cost-effectiveness study on SCID screening that models are sensitive to the cost of the test and that to maintain the cost at less than $\$ 100,000 /$ quality-adjusted year of life, the screening for SCID should cost less than $\$ 15$ per test. ${ }^{1}$ The cost per test using tandem mass spectrometry was less than $€ 0.01$.
It is without doubt that a method revealing all patients with SCID with a unique test would provide a greater benefit; however, at present, as reported by authors, even methods based on TREC analysis through molecular biology cannot individuate all known SCIDs and are undergoing refinement to improve specificity. ${ }^{1,15}$ The use of tandem mass spectrometry in the laboratories that are already performing neonatal screening programs can make possible a population-based newborn screening for ADA deficiency and can add diagnosis for one of the most frequent form of SCID at a negligible cost.

Clinical implications: The diagnosis of ADA-SCID can be performed at birth on DBS samples by using tandem mass spectrometry within a neonatal program of screening with no indeterminate or false-negative results and negligible cost.

\section{REFERENCES}

1. Puck JM. SCID Newborn Screening Working Group. Population-based newborn screening for severe combined immunodeficiency: steps toward implementation. J Allergy Clin Immunol 2007;120:760-8.

2. Buckley RH. Molecular defects in human severe combined immunodeficiency and approaches to immune reconstitution. Annu Rev Immunol 2004;22:625-55.

3. Notarangelo LD. Primary immunodeficiencies. J Allergy Clin Immunol 2010; 125(suppl 2):S182-94.

4. Sauer AV, Aiuti A. New insights into the pathogenesis of adenosine deaminasesevere combined immunodeficiency and progress in gene therapy. Curr Opin Allergy Clin Immunol 2009;9:496-502.

5. Hirschhorn R. Adenosine deaminase deficiency. Immunodefic Rev 1990;2:175-98.

6. Booth C, Hershfield M, Notarangelo L, Buckley R, Hoenig M, Mahlaoui N, et al. Management options for adenosine deaminase deficiency; proceedings of the EBMT satellite workshop (Hamburg, March 2006). Clin Immunol 2007;123: $139-47$

7. Sanchez JJ, Monaghan G, Børsting C, Norbury G, Morling N, Gaspar HB. Carrier frequency of a nonsense mutation in the adenosine deaminase (ADA) gene implies a high incidence of ADA-deficient severe combined immunodeficiency (SCID) in Somalia and a single, common haplotype indicates common ancestry. Ann Hum Genet 2007;71(suppl):336-47.

8. Gaspar BH, Aiuti A, Porta F, Candotti F, Hershfield MS, Notarangelo LD. How I treat ADA deficiency. Blood 2009;114:3524-32

9. Hershfield MS, Mitchell BS. Immunodeficiency diseases caused by adenosine deaminase deficiency and purine nucleoside phosphorylase deficiency. In: Scriver CR, Beaudet AL, Sly WS, Valle D, editors. The metabolic and molecular basis of inherited disease. New York: McGraw-Hill; 1995. p. 1725-68.

10. Chace DH. Mass spectrometry in newborn and metabolic screening: historical perspective and future directions. J Mass Spectrom 2009;44:163-70.

11. la Marca G, Malvagia S, Pasquini E, Innocenti M, Donati MA, Zammarchi E. Rapid 2nd-tier test for measurement of 3-OH-propionic and methylmalonic acids on dried blood spots: reducing the false-positive rate for propionylcarnitine during expanded newborn screening by liquid chromatography-tandem mass spectrometry. Clin Chem 2007;53:1364-9.

12. la Marca G, Malvagia S, Pasquini E, Innocenti M, Fernandez MR, Donati MA, et al. The inclusion of succinylacetone as marker for tyrosinemia type I in expanded newborn screening programs. Rapid Commun Mass Spectrom 2008;22:812-8.

13. Health Resources and Services Administration. Secretary's Advisory Committee on Heritable Disorders in Newborns and Children. Resolution from the 20th meeting, January 22, 2010; Washington DC. Available at: http://www.hrsa.gov/ heritabledisorderscommittee/. Accessed April 29, 2011.

14. Howell RR, Engelson G. Structures for clinical follow-up: newborn screening. J Inherit Metab Dis 2007;30:600-5.

15. Lipstein EA, Vorono S, Browning MF, Green NS, Kemper AR, Knapp AA, et al. Systematic evidence review of newborn screening and treatment of severe combined immunodeficiency. Pediatrics 2010;125:e1226-35.

16. Morgan G, Levinsky RJ, Hugh-Jones K, Fairbanks LD, Morris GS, Simmonds HA. Heterogeneity of biochemical, clinical and immunological parameters in severe combined immunodeficiency due to adenosine deaminase deficiency. Clin Exp Immunol 1987;70:491-9.

17. Hirschhorn R, Borkowsky W, Jiang CK, Yang DR, Jenkins T. Two newly identified mutations (Thr233Ile and Leu152Met) in partially adenosine deaminase-deficient (ADA-) individuals that result in differing biochemical and metabolic phenotypes. Hum Genet 1997;100:22-9. 
18. Santisteban I, Arredondo-Vega FX, Kelly S, Mary A, Fischer A, Hummell DS, et al. Novel splicing, missense, and deletion mutations in seven adenosine deaminase-deficient patients with late/delayed onset of combined immunodeficiency disease. Contribution of genotype to phenotype. J Clin Invest 1993;92: 2291-302.

19. Jenkins T. Red-blood-cell adenosine deaminase deficiency in a "healthy" Kung individual. Lancet 1973;2:736.

20. Davies PM, McBride MB, Simmonds HA. An improved screening method for inherited disorders of purine and pyrimidine metabolism by HPLC. Adv Exp Med Biol 1991;309B:7-10

21. Adam T, Friedecky D, Fairbanks LD, Sevcik J, Bartak P. Capillary electrophoresis for detection of inherited disorders of purine and pyrimidinemetabolism. Clin Chem 1999;45:2086-93.
22. Ito T, van Kuilenburg AB, Bootsma AH, Haasnoot AJ, van Cruchten A, Wada Y, et al. Rapid screening of highrisk patients for disorders of purine and pyrimidine metabolism using HPLC-electrospray tandem mass spectrometry of liquid urine or urine-soaked filter paper strips. Clin Chem 2000;46:445-52.

23. la Marca G, Casetta B, Malvagia S, Pasquini E, Innocenti M, Donati MA, et al. Implementing tandem mass spectrometry as a routine tool for characterizing the complete purine and pyrimidine metabolic profile in urine samples. J Mass Spectrom 2006;41:1442-52.

24. Azzari C, Massai C, Poggiolesi C, Indolfi G, Spagnolo G, De Luca M, et al. Cost of varicella-related hospitalisations in an Italian paediatric hospital: comparison with possible vaccination expenses. Curr Med Res Opin 2007;23:2945-54.

25. Demografia in cifre. Istituto Nazionale di Statistica, ISTAT. Available at: http:// demo.istat.it/. Accessed April 29, 2011.

\section{Did you know? The JACI has a new website!}

You can now personalize the $J A C I$ website to meet your individual needs. Enjoy these new benefits and more:

- Stay current in your field with Featured Articles of The Week, Articles in Press, and easily view the Most Read and Most Cited articles.

- Sign up for a personalized alerting service with Table of Contents Alerts, Articles in Press Alerts and Saved Search Alerts to notify you of newly published articles.

- Search across 400 top medical and health sciences journals online, including MEDLINE.

- Greater cross-referencing results from your online searches.

Visit www.jacionline.org today to see what else is new online! 